\title{
Financial Applications of the Mahalanobis Distance
}

\author{
Sebastian Stöckl ${ }^{1} \&$ Michael Hanke ${ }^{1}$ \\ ${ }^{1}$ Institute of Financial Services, University of Liechtenstein, Liechtenstein \\ Correspondence: Sebastian Stöckl, Institute of Financial Services, University of Liechtenstein, Liechtenstein
}

Received: September 10, 2014

Accepted: September 30, $2014 \quad$ Available online: October 23, 2014

doi:10.11114/aef.v1i2.511

URL: http://dx.doi.org/10.11114/aef.v1i2.511

\begin{abstract}
We describe existing and potential financial applications of the Mahalanobis distance. After a short motivation and a discussion of important properties of this multivariate distance measure, we classify its applications in finance according to the source and nature of its input parameters. Examples illustrate the usefulness of these applications of the Mahalanobis distance for financial market participants.
\end{abstract}

Keywords: Mahalanobis distance, financial turbulence, multivariate distance

\section{Introduction}

Many problems in finance, e.g., in asset allocation or risk management, involve multivariate random variables. Financial market participants are often interested in the similarity of realizations of such random variables. As an illustration, at some stage at the beginning of the sub-prime crisis practitioners began to realize that "the markets are different from what they used to be". Whereas at the beginning of the crisis this perception was confined to unusual behavior in prices of particular instruments like asset-backed securities, the effects quickly spread to other instruments and markets as the crisis unfolded. Despite undoubtedly processing "multivariate" information from different asset classes, regions, etc., many practitioners did not use a formal model to arrive at such conclusions. However, an objective indicator that previously stable relations between variables no longer hold would be desirable for many market participants: Portfolio weights optimized based on distributions that have changed may be far from optimal, and trading strategies trying to exploit market price regularities that are no longer present are prone to lose money.

A measure rooted in statistical theory and suitable to detect situations like these is the Mahalanobis distance. Developed by Mahalanobis (1927) for the classification of human skulls, it has since found numerous applications in many fields. Examples from finance include asset classification, portfolio surveillance and outlier detection. Closest in spirit to the questions described above, Kritzman and Li (2010) propose to use the Mahalanobis distance as a measure of "financial turbulence", which they understand as multivariate unusualness in financial market data. Given a vector of $n$ assets' means $\boldsymbol{\mu}$ and their covariance matrix $\Sigma$, they define financial turbulence as a property of a vector of these assets' returns $\boldsymbol{r}_{t}$ on a specific day $t$ via the squared Mahalanobis distance

$$
\mathrm{FT}_{t}=\left(\boldsymbol{r}_{t}-\boldsymbol{\mu}\right)^{\prime} \Sigma^{-1}\left(\boldsymbol{r}_{t}-\boldsymbol{\mu}\right)
$$

Generalizing this specific application based on the structure in eq. (1), we note that $\boldsymbol{r}_{t}, \boldsymbol{\mu}$ and $\Sigma$ may each be determined or estimated in various different ways. Depending on the source or nature of the data for each of these inputs, the resulting Mahalanobis distance will have a different economic interpretation. The aim of this paper is to explore some promising combinations of such choices and to discuss previous and potential uses of the resulting Mahalanobis distance measures together with their usefulness for financial market participants.

The paper is organized as follows: Some important properties of the Mahalanobis distance are described in Section 2. Section 3 discusses different input parameterizations appropriate for different types of applications, namely, sample-based differences, deviations from models, and forecast evaluation. Section 4 concludes.

\section{The Mahalanobis Distance}

The initial motivation for developing the Mahalanobis distance measure was to analyze and classify human skulls into groups, based on various properties (Mahalanobis, 1927). In a financial context, such properties could be the moments of assets in a portfolio, or of the portfolios on the balance sheet of an investment company. In this section, we will provide some background on the Mahalanobis distance. Using the financial turbulence measure of Kritzman and Li 
(2010) as an example, we provide an intuitive justification of the Mahalanobis distance together with the required assumptions, before discussing further properties which are useful in the context of finance.

When used as suggested by Kritzman and Li (2010), the Mahalanobis distance can be interpreted as an indicator of unusualness in financial markets. Assume an investor observes an asset's return $r_{t}$ on a specific day $t$. One way to assess the (standardized) magnitude of its deviation from usual is to relate the squared difference from its expected return $\mu$ to the asset's return variance: $\left(r_{t}-\mu\right)^{2} / \sigma^{2}$. The higher the resulting ratio, the more "unusual" is the level of $r_{t}$, in the sense that the instantaneous return variance exceeds its long-term average. This ratio, however, is exactly the definition of the squared Mahalanobis distance for one asset. What would then be the best way to generalize this measure to higher dimensions? A simple extension would be to add the Mahalanobis distances of all $n$ assets in a portfolio:

$$
\mathrm{Eu}_{t}^{2}=\sum_{i=1}^{n} \frac{\left(r_{t, i}-\mu_{i}\right)^{2}}{\sigma_{i}^{2}}
$$

which is the squared Euclidean distance. This, however, would completely neglect any dependence between the assets. The direction of the deviation of asset returns from their means contains additional information, which should also be captured by a multivariate distance measure. Assume two highly correlated assets, whose returns show large positive deviations from their means on a given day. On the next day, both returns are at the same distance from their means, but one return is still above its mean, whereas the other one is now below its mean. The measure defined in eq. (2) would yield exactly the same deviation as in the first case, but for highly correlated assets it is even more unusual to deviate from their means in opposite directions. The squared Mahalanobis distance takes this into account:

$$
\mathrm{Ma}_{t}^{2}=\left(\boldsymbol{r}_{t}-\boldsymbol{\mu}\right)^{\prime} \Sigma^{-1}\left(\boldsymbol{r}_{t}-\boldsymbol{\mu}\right)
$$

Note that eq. (3) reduces to eq. (2) if the covariance matrix is diagonal (i.e., off-diagonal elements are equal to zero). Hence, as a measure of unusualness in financial markets, the main advantage of the Mahalanobis distance compared to classical risk measures is its ability to capture deviations in the joint movements of asset returns $r_{i}$ and $r_{j} \quad(i, j=$ $1, \ldots, n ; i \neq j$ ). It condenses the information on unusual behavior across all assets into one single number.

The Mahalanobis distance does not require strong distributional assumptions: It is an appropriate measure of multivariate distance for elliptically distributed random variables (Note ${ }^{\mathrm{i}}$ ), which are completely described by their location parameter $\boldsymbol{\mu}$ and scatter matrix $\Sigma$. This leads to a nice geometric interpretation: All realizations with the same Mahalanobis distance are located on an ellipsoid whose position and shape is completely determined by $\boldsymbol{\mu}$ and $\Sigma$ (Meucci, 2009; Geyer, Hanke \& Weissensteiner, 2014).

In addition to these features, the Mahalanobis distance has several useful statistical properties. If asset returns are multivariate normal $r_{t} \sim N_{n}(\mu, \Sigma)$, the squared Mahalanobis distance is $\chi^{2}(n)$ distributed with $n$ degrees of freedom. For sample moments, the distribution of the squared Mahalanobis distance is a scaled beta distribution (Note ${ }^{\text {ii }}$ ) that converges towards the $\chi^{2}(n)$ with increasing sample size $N$. Additionally, the Mahalanobis distance defines a metric that is invariant under affine transformations (scale and translation) $Y=a+B \cdot X$ (Meucci, 2009), and thus the scaling of the asset returns is irrelevant.

For some applications, this transformation invariance may lead to undesirable results of the "raw" Mahalanobis distance as defined in eq. (3). For instance, the Mahalanobis distance for a portfolio should react less strongly if the assets that show unusual behavior have only small weights in the portfolio. A natural remedy is to adjust the distance measure to account for portfolio weights, which results in a squared, weighted and normalized Mahalanobis distance. Since this measure is particularly useful in the context of portfolios, we call it (squared) portfolio turbulence:

$$
\operatorname{PFT}_{t}^{2}=\frac{1}{w^{2}}\left(w_{D}\left(\boldsymbol{r}_{t}-\boldsymbol{\mu}\right)\right)^{\prime} \Sigma^{-1}\left(w_{D}\left(\boldsymbol{r}_{t}-\boldsymbol{\mu}\right)\right),
$$

where $w_{D}$ is the diagonal matrix of weights $w_{i}$. Portfolio turbulence is distributed as generalized $\chi^{2}$ with parameters $\Sigma$ and $w_{D} \Sigma^{-1} w_{D}$ (Jones, 1983) with an expectation of 1 (due to the normalization factor $\boldsymbol{w}^{2}:=\operatorname{tr}\left(w_{D}^{2}\right)$ ). (Note ${ }^{\text {iii }}$ )

\section{Types of Financial Applications}

In this section, we will categorize different types of financial applications for the Mahalanobis distance, based on the source and nature of the input parameters used.

\subsection{Sample-Based Differences}

One type of applications relates to comparisons of financial variables (e.g., asset returns) observed in different time periods. An example for this is the "markets are different now" statement addressed in the introduction. In many situations investors would benefit from knowing that previously stable relations in market variables no longer prevail. Ex post (i.e., some time after the event), such a change could be detected using standard tests for structural breaks in time series. For these tests to have enough power, quite a large number of observations after the change are required. Therefore, they might be too late in indicating that such a break has occurred. Using past values over an extended time period to estimate $\boldsymbol{\mu}$ and $\Sigma$, the Mahalanobis distance would already be high on the first day of "unusual" returns, and successive high values would indicate (at least temporal) deviations in the behavior of market variables. 
We distinguish two types of sample-based differences: Either we compare a particular realization to a historical sample, or we compare two samples. The first type of comparison is in the sense of financial turbulence (Kritzman \& Li, 2010) or portfolio turbulence as introduced in eq. (4). It allows for a comparison of today's returns to their historical means $(\boldsymbol{\mu}=\overline{\boldsymbol{x}})$ and sample covariances $(\Sigma=S)$ either across assets and markets, or within a portfolio. When used in this way, the Mahalanobis distance is useful, e.g., for market and portfolio surveillance. Values above a certain (statistically determined) threshold indicate to market participants potential structural changes in markets (i.e., the switch from nonturbulent to turbulent markets), which might require adjustments in portfolio weights or a reduction in exposure of the overall portfolio. (Note ${ }^{\text {iv }}$ )

The second type of sample-based applications using the Mahalanobis distance is the comparison of two samples, e.g., returns of the same set of assets over different time periods. Given a (exogenously determined) partition of the time interval $[0, T]$ into several subperiods $\tau_{1}, \ldots, \tau_{m}$ (with corresponding return means $\overline{\boldsymbol{x}}_{k}$ and covariances $\Sigma_{k}$, $k=1, \ldots, m$ ), asset returns in subperiods $\tau_{k}$ and $\tau_{l}$ can be compared independently of the (obvious) inherent dependence/correlation of the returns by building a combined covariance matrix $\Sigma_{k, l}(k \neq l)$ according to McLachlan (1999). (Note ${ }^{v}$ ) The Mahalanobis distance can also be used to indicate the beginning and the end of turbulent and nonturbulent periods, to compare the parameters of those subsequent periods. Such an approach is suggested by Chow, Jacquier, Kritzman and Lowry (1999), who, for purposes of risk management, advocate the use of parameters estimated in turbulent periods, in contrast to robust estimation procedures for mean and variance, where outliers are detected (often using the Mahalanobis distance) and eliminated. (Note ${ }^{\mathrm{vi}}$ )

Example 1. An intuitive example for this type of application is the change in market conditions during the financial crisis. Assume an investor whose portfolio consists of four major asset classes: $3 / 8$ of his wealth is in equities, $1 / 4$ in fixed income, $1 / 4$ in real estate, and 1/8 in alternative investments. The investor observes the descriptive statistics for the period of 2004-2006, given in Table 1.

Table 1.

\begin{tabular}{lllllllll}
\hline 2004-2006 & mean & std.dev & skewness & ex.kurtosis & correlations & & \\
\hline Equities & 0.0006 & 0.0058 & -0.23 & 1.02 & 1.00 & 0.32 & 0.72 & 0.11 \\
Fixed income & 0.0001 & 0.0035 & 0.00 & 1.01 & 0.32 & 1.00 & 0.39 & 0.12 \\
Real estate & 0.0010 & 0.0067 & -0.49 & 1.40 & 0.72 & 0.39 & 1.00 & 0.04 \\
Alt. investments & 0.0003 & 0.0146 & 0.13 & 0.43 & 0.11 & 0.12 & 0.04 & 1.00 \\
\hline
\end{tabular}

Descriptive statistics (daily data) for the asset classes in Example 1. Time frame: 01/2004-12/2006. We used the following indices as proxies for these asset classes: Equities: FTSE ALL WORLD, Fixed income: Barclays Multiverse All, Real estate: FTSE EPRA/NAREIT Global, Alt. investments (proxied using commodities): S\&P GSCI Commodities. Datasource: Thomson Reuters Datastream.

Based on the observed $\boldsymbol{\mu}$ and $\Sigma$, the investor calculates the portfolio turbulence defined in eq. (4) to get an indication whether market conditions have changed substantially compared to the estimation period (2004-2006), which might induce him to re-assess his asset allocation. The results are depicted in Figure 1. The figure also includes $\mu \pm \sigma$-confidence bands at values of 0.1611 and 1.8389 , respectively. From the second half of 2007 onwards, values exceeding the confidence band increase both in frequency and in their average level, which can be seen from the 30-day moving average (blue line). In Jan. 2008, the $\mu+\sigma$ confidence band is exceeded repeatedly within a short period of time providing clear signs that markets have changed substantially. Around the Lehman Brothers event in September 2008, a high percentage of portfolio turbulence values are outside the confidence interval, and so is the 30-day moving average.

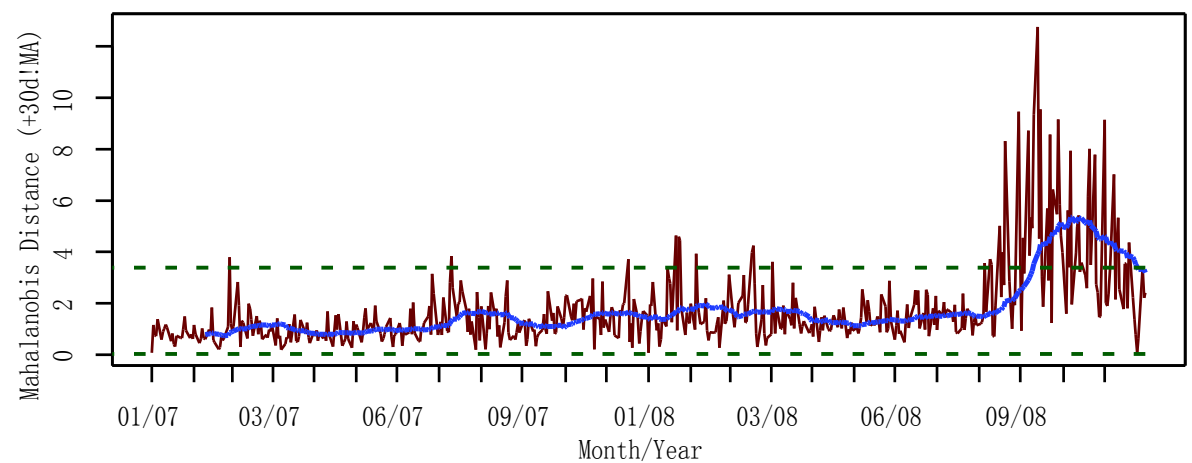

Figure 1. Daily portfolio turbulences (weighted Mahalanobis distances between realized returns and historical returns), parameter sample period 01/2004-12/2006, with their 30-day moving average (blue line) and $\mu \pm \sigma$-confidence bands of the portfolio described in Example 1. 


\subsection{Deviations from Model Prices and Checks for Arbitrage Opportunities}

Asset pricing models, both those theoretically motivated (like the CAPM) and those rather empirically driven (like the Fama-French three-factor model), imply certain return distributions. The Mahalanobis distance can also be applied to assess deviations between observed asset returns and those implied theoretically. Whereas in Section 3.1 observed returns were compared to empirical asset return distributions (without any reference to financial theory), this type of application asks how well current observations are in line with particular models. Deviations from model-implied distributions can be a useful indicator for bubbles in markets. Also, when applied to several competing models, predominantly lower Mahalanobis distances for one of these models could be taken as an indication that (among the candidate models) this model best describes what we observe in markets.

Many asset pricing models imply a multivariate normal distribution. When parameterized with theoretically derived values for $\boldsymbol{\mu}$ and $\boldsymbol{\Sigma}$, this allows for an evaluation according to eq. (3). Compared to the description of the first type of sample-based differences in Section 3.1 (where we compared current values to historical averages), the parameters defining such a reference distribution could be updated more frequently to changed market conditions. Comparing to this reference distribution may be more desirable than comparisons with historical data, which may no longer be considered as representative. An example for theory-based derivation of distribution parameters is the inversion of the CAPM according to Black and Litterman (1992).

Even without any distributional assumptions (Note vii), Geyer et al. (2014) show that the Mahalanobis distances of discrete samples (scenarios in portfolio optimization and risk management) of expected returns for a given covariance matrix determine areas of (potential) possibilities of arbitrage. These areas (ellipsoids) allow to determine whether a scenario is arbitrage free, admits arbitrage (in which case it is useless and must be discarded), or requires additional testing.

Example 2. The Black and Litterman (1992) model is a well-known combination of theory (CAPM) and observed data to arrive at implied asset return distributions. Deviations of current observations from these implied distributions can be interpreted as unusual pricing behavior relative to theory (as opposed to the purely empirical approach in Example 1 in Section 3.1). We illustrate this on a dataset of 22 country indices observed and aggregated between 07/1988 and 12/2012 (cf. Kaiser, Menichetti \& Veress, 2013).

Implied expected returns are computed as $\boldsymbol{\mu}_{t+1}=\delta \Sigma_{t} \boldsymbol{w}_{t}$ (cf. Walters, 2011) where $\delta$ denotes the risk aversion parameter (for our illustrative example, we assume $\delta=1$ ), and $\boldsymbol{w}_{t}$ is the vector of weights derived from the average market capitalizations of the indices during the previous month. The covariance matrix $\Sigma_{t}$ is estimated based on a 120-month rolling window. We can then calculate the Mahalanobis distances of realized returns $\boldsymbol{r}_{t}$ from their implied expectations $\boldsymbol{\mu}_{t}$ using the implied expectations' covariance matrix $\Sigma_{\mu t}=\tau \Sigma_{t}$ (we set $\tau=1$, cf. Walters, 2011). The results are shown in Figure 2. Spikes that are easily identified include the Russian crisis in 1998, the burst of the tech-stock bubble in 2000, and the Lehman event in the second half of 2008.

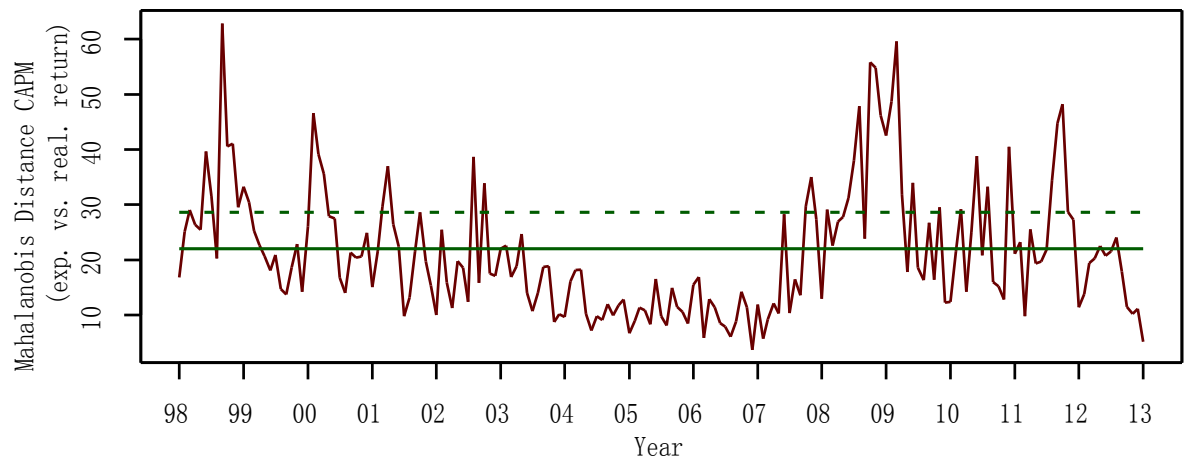

Figure 2. Squared Mahalanobis distances of realized vs. Black/Litterman-implied values, together with their mean (solid horizontal line) and the corresponding upper one standard deviation band (dashed horizontal line) to determine values that deviate strongly from those implied by theory (the CAPM).

\subsection{Forecast Evaluation}

Another domain where the Mahalanobis distance can provide valuable information is in the evaluation of forecasts. Here, $\boldsymbol{\mu}_{t}$ represents a multivariate point forecast from a prediction model, whose quality is to be evaluated using (possibly successive) observations $\boldsymbol{r}_{t}$. Choosing the mean squared error (MSE) as the loss function, the MSE-matrix determines the shape of the confidence ellipsoid (defined by the Mahalanobis distance) centered at $\boldsymbol{\mu}_{t}$. The lower the 
Mahalanobis distance between observation and prediction, the better the forecast. Successive high values of the Mahalanobis distance could be taken as an indication that a previously reliable forecasting model no longer works as well as it did in the past, raising a warning flag for the user of the model.

In this section we distinguish two such approaches based on the Mahalanobis distance. Jordà and Marcellino (2010) use the Mahalanobis distance to calculate forecast regions for a path of forecasts from periods 1 to $H$. Such pathwise forecasts are important, because many financial time series are autocorrelated and require adjustable confidence regions for their predictions. (Note ${ }^{\text {viii }}$ )

The second application relates to a multivariate point-forecast, where we do not want to evaluate the forecast across time given one variable, but evaluate the forecast across all variables at a specified forecast horizon. Forecast regions in the form of ellipsoids are again constructed based on the Mahalanobis distance (e.g., Lütkepohl, 2006, p. 40 and Appendix B.2).

Example 3. We illustrate the use of the Mahalanobis distance in forecast evaluation using an example from FX forecasting based on Zivot and Wang (2006). Denote by $\mathrm{d} s_{t}=\Delta \log \left(s_{t}\right)$ the log differences of the USD/GBP spot rate, and by $f p_{t}=\log \left(f_{t}\right)-\log \left(s_{t}\right)$ the 30-day $\log$ forward premium. Both are observed from 01/1980 to 07/2013. After testing for and confirming stationarity of the series (Augmented-Dickey-Fuller-tests at the 1\%-level), we model both series using a vector-autoregressive process of order 1 , where the lag order is chosen according to the Schwartz criterion.

Estimating this model for the time period 01/1980-05/2013 yields the following process:

$$
\left(\begin{array}{l}
\mathrm{d} s_{t} \\
f p_{t}
\end{array}\right)=\left(\begin{array}{l}
-0.0037^{*} \\
-0.0001^{*}
\end{array}\right)+\left(\begin{array}{cc}
0.0614 & -1.9710^{* * *} \\
0.0006 & 0.9187^{* * *}
\end{array}\right)\left(\begin{array}{l}
\mathrm{d} s_{t-1} \\
f p_{t-1}
\end{array}\right)+\left(\begin{array}{l}
u_{1 t} \\
u_{2 t}
\end{array}\right)
$$

with residual covariance (MSE) matrix $\Sigma_{u}$ (see Table 2). (Note ${ }^{\text {ix }}$ ) The predictions and the MSE-matrices $\Sigma_{y}(h)$ $\left(\right.$ Note $^{\mathrm{x}}$ ) for 06/2013-08/2013 are shown in Table 2, together with their realized values.

Table 2.

\begin{tabular}{|c|c|c|c|c|c|c|}
\hline Forecasts & $\mathrm{d} s_{t+h}$ & $f p_{t+h}$ & $\mathrm{~d} s_{t}(h)$ & $f p_{t}(h)$ & & \\
\hline $06 / 2013$ & 0.0004 & -0.0002 & -0.0049 & -0.0003 & & \\
\hline $07 / 2013$ & -0.0002 & -0.0002 & -0.0034 & -0.0004 & & \\
\hline $08 / 2013$ & & & -0.0031 & -0.0005 & & \\
\hline $\operatorname{MSE} \sum_{\mathrm{y}}(\mathrm{h})$ & 06/2013 & & $07 / 2013$ & & $08 / 2013$ & \\
\hline \multirow{2}{*}{$10-5$} & 88.15 & -0.24 & 88.79 & -0.37 & 89.75 & -0.16 \\
\hline & -0.24 & 0.06 & -0.37 & 0.12 & -0.16 & 0.16 \\
\hline
\end{tabular}

Forecasts $(06 / 2013-08 / 2013)$ for the log return of the USD/GBP spot rate $\left(\mathrm{d}_{s_{t}}(h)\right)$ and the forward premium $\left(f p_{t}(h)\right)$, using the VAR process in equation (5). In addition, it shows the realized values $\mathrm{d} s_{t+h}$ and $f p_{t+h}$ (in-sample estimates for 06/2013 and 07/2013) for these variables together with all corresponding MSE matrices. Datasource: Bank of England.

Following Lütkepohl (2006, p.40), we can now calculate the joint forecast confidence ellipse per time step. Figure 3 shows confidence ellipses for three forecasts, the first two of them together with their realized values $(06 / 2013$, solid line and 07/2013, dashed), the last one as an out-of-sample forecast (08/2013, dotdashed). The notorious difficulty in predicting exchange rates causes the confidence ellipses to grow rather quickly.

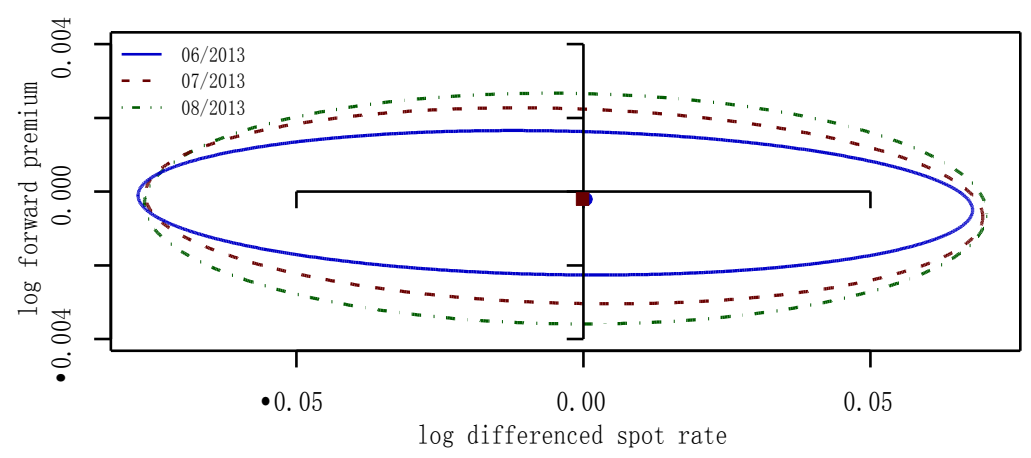

Figure 3. Forecast confidence ellipses for two in-sample (06/2013, solid, and 07/2013, dashed) and one out-of-sample (08/2013, dotdashed) forecast, based on the Mahalanobis distance 


\section{Conclusion}

Given that most problems in finance are multivariate in nature, the Mahalanobis distance lends itself naturally to questions regarding the existence and magnitude of multivariate deviations (i) between observations, (ii) between observations and theoretically implied values, or (iii) between observations and predictions, to name just a few of the possible applications. Depending on the source and nature of the data used as inputs, the economic interpretation of the resulting distance measure differs, making it a versatile measure with a solid statistical foundation.

\section{References}

Black, F., \& Litterman, R. (1992). Global portfolio optimization. Financial Analysts Journal 48, $28-43$. http://dx.doi.org/10.2469/faj.v48.n5.28.

Bodnar, O. (2009). Sequential surveillance of the tangency portfolio weights. International Journal of Theoretical and Applied Finance 12, 797-810. http://dx.doi.org/10.1142/S0219024909005464.

Cambanis, S., Huang, S., \& Simons, G. (1981). On the theory of elliptically contoured distributions. Journal of Multivariate Analysis 11, 368-385. http://dx.doi.org/10.1016/0047-259X(81)90082-8.

Chow, G., Jacquier, E., Kritzman, M., \& Lowry, K. (1999). Optimal portfolios in good times and bad. Financial Analysts Journal, 55, 65-73. http://dx.doi.org/10.2469/faj.v55.n3.2273.

Geyer, A., Hanke, M., \& Weissensteiner, A. (2014). No-arbitrage bounds for financial scenarios. European Journal of Operational Research, 236, 657-663. http://dx.doi.org/10.1016/j.ejor.2014.01.027.

Golosnoy, V. (2007). Sequential monitoring of minimum variance portfolio. Advances in Statistical Analysis, 91, 39-55. http://dx.doi.org/10.1007/s10182-006-0016-8.

Jones, D. A. (1983). Statistical analysis of empirical models fitted by optimization. Biometrika, 70, 67-88. http://dx.doi.org/10.1093/biomet/70.1.67.

Jordà, Ò., \& Marcellino, M. (2010). Path forecast evaluation. Journal of Applied Econometrics, 25, 635-662. http://dx.doi.org/10.1002/jae.1166.

Kaiser, L., Menichetti, M. J., \& Veress, A. (2014). Enhanced Mean-Variance Portfolios - A Controlled Integration of Quantitative Predictors. Journal of Portfolio Management, 40(4), 28-41. http://doi.org/10.3905/jpm.2014.40.4.028.

Kritzman, M., \& Li, Y. (2010). Skulls, financial turbulence, and risk management. Financial Analysts Journal, 66, 30-41. http://dx.doi.org/10.2469/faj.v66.n5.3.

Lütkepohl, H. (2006). New introduction to multiple time series analysis. Berlin: Springer.

Mahalanobis, P. C. (1927). Analysis of race-mixture in Bengal. Journal of the Asiatic Society of Bengal, 23, $301-333$.

Mardia, K. V., Kent, J. T., \& Bibby, J. M. (1979). Multivariate analysis. London: Academic Press

Mathai, A. M., \& Provost, S.B. (1992). Quadratic Forms in Random Variables: Theory and Applications. New York: Marcel Dekker Incorporated.

McLachlan, G. J. (1999). Mahalanobis distance. Resonance, 4, 20-26. http://dx.doi.org/10.1007/BF02834632.

Meucci, A. (2009). Risk and Asset Allocation (1st ed.). New York: Springer.

Mitchell, A.F., \& Krzanowski, W.J. (1985). The mahalanobis distance and elliptic distributions. Biometrika, 72, $464-467$. http://dx.doi.org/10.1093/biomet/72.2.464.

Owen, J., \& Rabinovitch, R. (1983). On the class of elliptical distributions and their applications to the theory of portfolio choice. The Journal of Finance, 38, 745-752. http://dx.doi.org/10.1111/j.1540-6261.1983.tb02499.x.

Rao, C. R. (2009). Linear Statistical Inference and its Applications. New York: Wiley.

Walters, J. (2011). The Black-Litterman Model in Detail. SSRN Scholarly Paper ID 1314585. http://papers.ssrn.com/abstract=1314585.

Zivot, E., \& Wang, J. (2006). Modeling financial time series with S-plus. New York: Springer.

\section{Notes}

Note ${ }^{i}$. For the definition and properties of elliptical distributions see Cambanis, Jacquier, Kritzman and Lowry (1981), Mitchell and Krzanowski (1985), and for their connection to portfolio theory see Owen and Rabinovitch (1983).

Note ${ }^{\text {ii }} \cdot \frac{N}{N^{2}-1} \operatorname{Ma}_{t}^{2}(\bar{r}, S) \sim \operatorname{Beta}\left(\frac{n}{2}, \frac{N-n-1}{2}\right)$, see Mardia, Kent and Bibby (1979). 
Note ${ }^{\text {iii. }}$. For general $Y \sim N_{n}(\mu, \Sigma)$ and symmetric $n \times n$-matrix $A$, we have $Q=Y^{\prime} A Y$ with $E[Q]=\operatorname{tr}(A \Sigma)+\mu^{\prime} A \mu$ and $\operatorname{Var}[Q]=$ $2 \operatorname{tr}(A \Sigma A \Sigma)+4 \mu^{\prime} A \Sigma A \mu$ (Mathai \& Provost, 1992). Given $\left(\boldsymbol{r}_{t}-\boldsymbol{\mu}\right) \sim N_{n}(0, \Sigma), A=w_{D} \Sigma^{-1} w_{D}$ implies for the weighted and squared Mahalanobis distance $Q=\left(w_{D}\left(\boldsymbol{r}_{T}-\boldsymbol{\mu}\right)\right)^{\prime} \Sigma^{-1}\left(w_{D}\left(\boldsymbol{r}_{T}-\boldsymbol{\mu}\right)\right)$ that $E[Q]=\operatorname{tr}\left(w_{D}^{2}\right)=: \boldsymbol{w}^{2}$ and $\operatorname{Var}[Q]=2 \operatorname{tr}\left(w_{D}^{4}\right)$, and thus $E\left[\mathrm{PFT}_{t}^{2}\right]=1$ and $\operatorname{Var}\left[\mathrm{PFT}_{t}^{2}\right]=\frac{2}{w^{2^{2}}} \operatorname{tr}\left(w_{D}^{4}\right)$.

Note ${ }^{\text {iv }}$. Golosny (2007) and Bodnar (2009) give estimates on the changes of the minimum variance as well as the tangency portfolio weights with respect to distributional changes in the asset returns detected using the Mahalanobis distance.

Note ${ }^{\mathrm{v}}$. This is exactly the Hotelling $T^{2}$-test for multivariate dependent samples, making use of the squared Mahalanobis distance (Rao, 2009).

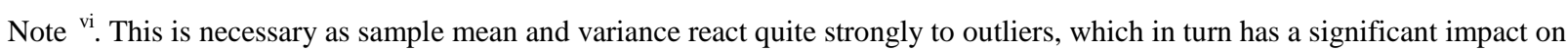
portfolios estimated using those parameters (Bodnar, 2009).

Note vii . Except for the existence of the first two moments of the multivariate return distribution.

Note ${ }^{\text {viii }}$. The Wald statistic that is used to calculate those forecast regions corresponds to the Mahalanobis distance. For multivariate paths, the forecast regions are determined separately for each component variable.

Note ${ }^{\text {ix }}$. Significance levels are provided with the coefficients using standard notation. R-Squares (adjusted) for both equations are $0.0240(0.191)$ and $0.8424(0.8416)$, respectively.

Note $^{\mathrm{x}} . \Sigma_{y}(1)=\Sigma_{u}$ and $\Sigma_{y}(h+1)=\Sigma_{y}(h)+\Phi_{h} \Sigma_{y}(h) \Phi_{h}^{\prime}$, cf. Lütkepohl (2006).

\section{(cc) EY}

This work is licensed under a Creative Commons Attribution 3.0 License. 